\title{
Actin and Rho GTPases in herpesvirus biology
}

\author{
Herman W. Favoreel ${ }^{1}$, L.W. Enquist ${ }^{2}$ and Becket Feierbach ${ }^{2}$ \\ ${ }^{1}$ Department of Virology, Parasitology, and Immunology, Faculty of Veterinary Medicine, Ghent University, \\ 9820 Merelbeke, Belgium \\ ${ }^{2}$ Department of Molecular Biology, Princeton, NJ 08544, USA
}

\begin{abstract}
Viruses have evolved a variety of interactions with host cells to create an optimal niche for viral replication, persistence and spread. The actin cytoskeleton of the host cell and actin-regulating Rho GTPase signaling pathways can be involved in several of these interactions. This review focuses on recent findings on herpesvirus interactions with actin and Rho GTPases during viral entry, replication in the nucleus and egress. Unraveling these often fascinating interactions might also provide additional insights into sometimes poorly known aspects of actin biology (e.g. its role in the nucleus) and in the development of novel antiviral therapies.
\end{abstract}

\section{Actin filaments and actin-regulating Rho GTPase signaling pathways}

Filamentous actin is a key structure of the cytoskeleton in every cell. The actin cytoskeleton is involved in many crucial cellular processes, including providing cell integrity, mobility and shape; driving cell division and contraction; and the uptake of extracellular molecules.

Actin filaments consist of polarized ATP-bound globular actin monomers. These filamentous actin (F-actin) molecules can associate into bundles or networks through actin cross-linking molecules. The best characterized F-actin bundles and networks are filopodia, lamellipodia and actin stress fibers. Formation of the different actin bundles and networks is regulated by signal transduction pathways that depend on small Rho GTPases [1]. Of the 22 identified mammalian Rho GTPases, the best characterized are RhoA (stress fibers), Rac1 (lamellipodia) and Cdc42 (filopodia). These Rho GTPases act as molecular switches and alternate between their active GTP-bound form and their inactive GDP-bound form. Transition from the active to the inactive form is accompanied by phosphorylation and activation of different downstream effector molecules that initiate signal transduction pathways and subsequent rearrangements in the actin cytoskeleton. These three signal transduction pathways are interwoven and the RhoA pathway generally counteracts the Rac1 and Cdc42 pathways [2]. Details of Rho GTPase signaling networks can be found elsewhere [3].

Because of the many cellular functions in which they are involved, it comes as no surprise that many intracellular

Corresponding author: Favoreel, H.W. (herman.favoreel@UGent.be)

Available online 30 August 2007. pathogens interact with actin and actin-regulating signaling pathways. These interactions can occur at several points in the life cycle of the pathogens, including, although not limited to, internalization, intracellular movement and intercellular spread [4,5].

There are recent indications that, in particular cell types, herpesviruses also interact with actin and/or Rho GTPases during three major phases of the replication cycle of herpesviruses: entry into the cytoplasm, replication and assembly in the nucleus and maturation and egress. This review presents an overview of these data and points to future directions in research in this new and potentially important field of herpesvirus biology. Special attention is given on the alphaherpesvirus subfamily of herpesviruses, but relevant references to work on other herpesviruses are also mentioned. Alphaherpesviruses are the largest subfamily of the Herpesviridae and comprise many closely related pathogens, including herpes simplex virus (HSV) and varicella-zoster virus (VZV) in humans and several animal pathogens, such as pseudorabies virus (PRV) in pigs, bovine herpesvirus 1 in cattle, equine herpesvirus 1 in horses and Marek's disease virus (MDV) in chicken.

\section{Virus entry in host cells}

Cell-type-dependent involvement of actin in herpesvirus entry

Following uptake in host cells, herpesviruses travel to the nucleus through microtubule-based transport (reviewed in $[6,7])$. A direct role for actin in herpesvirus entry is less obvious and seems to be cell-type dependent.

Alphaherpesviruses have been thought generally to enter cells by direct fusion of the viral envelope with the plasma membrane of a host cell. Such direct fusion occurs in specific cell lines, such as Vero cells [8]. Inhibition of actin polymerization in Vero cells has little or no effect on virus entry, indicating that this type of entry does not depend on actin directly $[9,10]$. However, accumulating evidence suggests alternative $\mathrm{pH}$-dependent and -independent endocytic entry routes for alphaherpesviruses in a variety of cell types, including primary human corneal fibroblasts, human epidermal keratinocytes, C10 murine melanoma cells, Chinese hamster ovary (CHO) cells and HeLa cells [11-14]. A recent report indicates a role for actin in one of these alternative entry pathways. Clement and coworkers found that HSV-1 entry in primary human corneal fibroblasts and nectin-1-overexpressing $\mathrm{CHO}$ cells is associated with formation of actin-based filopodia and 
subsequent uptake of the virus particles through a phagocytosis-like process [12]. In addition, cytochalasin D and latrunculin $\mathrm{B}$, two inhibitors of actin polymerization, blocked uptake of the virus efficiently [12]. The dependence of herpesviruses on actin polymerization for successful entry might therefore rely on the entry route taken by the virus in a particular cell type.

\section{Rho GTPase signaling pathways during herpesvirus entry}

The viral $\mathrm{gD}$ envelope protein is essential for stable binding and for entry of nearly all alphaherpesviruses (except VZV and MDV). Remarkably, this single protein interacts with one of several cellular receptors, including nectin-1, nectin-2, herpes virus entry mediator and modified heparan sulfate [15]. Nectins are part of the immunoglobulin receptor family and are of paramount importance in gD-mediated herpesvirus entry into several cell types. They constitute cell adhesion molecules that are associated with the actin cytoskeleton through a molecule called afadin. In addition, they activate Cdc42 and Rac1, which results in the induction of filopodia and lamellipodia in epithelial cells and fibroblasts and in the formation of synapses in neurons $[16,17]$.

Using L cells expressing nectin variants unable to bind afadin, Sakisaka and colleagues demonstrated that the direct physical association of nectins with the actin cytoskeleton is superfluous for herpesvirus entry [18]. However, there is some evidence for nectin-mediated activation of Rho GTPase signaling during herpesvirus entry, although the biological significance is still largely unknown.

De Regge and colleagues reported that interaction of the gD envelope protein with nectin-1 (and possibly other nectins) during entry of the swine alphaherpesvirus PRV in sensory neurons of the trigeminal ganglion, a predominant site for herpesvirus latency, resulted in the Cdc42-driven formation of synaptic boutons [19] (Figure 1). Similar gD- and nectin-dependent formation of synaptic boutons has been reported for recombinant HSV-1 gD in rat hippocampal neurons [17]. Synaptic boutons have been described to represent sites of herpesvirus egress from axons [19-21], suggesting a possible role for $\mathrm{gD}$ - and nectin-induced synaptic boutons in intercellular viral spread.

In support of Cdc42 signaling during alphaherpesvirus entry, entry of HSV-1 into primary corneal fibroblasts, Madin-Darby canine kidney II (MDCKII) cells and nectin-1-overexpressing $\mathrm{CHO}$ cells results in a temporary activation of Cdc42 [12,22]. In nectin-1-overexpressing $\mathrm{CHO}$ cells, brief Cdc42 activation was accompanied with a more extended RhoA activation and the induction of filopodia-like structures within 30 min of virus attachment [12] (Figure 1). Virus particles associated preferentially with these filopodia, possibly facilitating 'surfing' on the plasma membrane, an actin-myosin-driven transport process that has been described to precede cellular entry of different viruses [23]. The underlying reason why herpesviruses might induce filopodia-like structures on virus binding to cellular receptors remains unclear. Although speculative, it might be that these filopodia enhance subsequent endocytic or phagocytic uptake of herpesvirus particles [12] or that, by inducing these virus-capturing structures on cells that display the appropriate virus receptors, additional virus particles are recruited to increase the chance of successful infection.

Hoppe and colleagues suggested a role for Cdc42 in herpesvirus entry by over-expressing Cdc42 mutants in MDCKII cells, finding decreased infectivity [22]. These studies suggested that inhibition of viral infectivity occurred after the virus had crossed the plasma membrane and thus after any virus surfing had taken place (Figure 1).

Alphaherpesvirus entry can be associated with Cdc42driven processes, but there is not a consensus on the activation of Rac 1 and RhoA signaling during entry. There is evidence for Rac1 activation but not RhoA activation during HSV-1 entry in MDCKII cells and vice versa in primary corneal fibroblasts and nectin-1 over-expressing CHO cells [12,22]. One possible explanation for this difference in signaling might again be the mode of viral entry. In CHO cells and primary corneal fibroblasts, Rho GTPase signaling is associated with a newly described phagocytosis- or micropinocytosis-like $\mathrm{pH}$-dependent entry route of HSV particles [12,24] (Figure 1). It is noteworthy that phagocytic uptake of HSV is associated with activation of RhoA and Cdc42 but not Rac1 [12]. Two common types of phagocytosis have been described: complement receptor 3-mediated phagocytosis (associated with little membrane protrusion and ruffling activity and reliant on Rho activation) and Fcy receptor-mediated phagocytosis (characterized by extensive filopodia and lamellipodia formation and requires Rac1 and Cdc42 activation) [25]. Although speculative, this possibly suggests that a cell-type-specific intermediate pathway is engaged during HSV entry.

Rho GTPase signaling might be a common feature of herpesvirus entry because it also occurs during entry of the gammaherpesvirus Kaposi's sarcoma-associated herpesvirus (HHV-8). The interaction between the HHV-8 gB envelope protein and $\alpha_{3} \beta_{1}$ integrin receptors resulted in induction of signaling through the RhoA, Rac1 and Cdc42 GTPases $[26,27]$. Activation of Rho GTPases during HHV-8 entry results in a profound reorganization of the actin architecture but also results in increased acetylation and stabilization of microtubules [27,28]. Interestingly, this increased acetylation and stabilization, rather than the alterations in the actin architecture, seems to facilitate microtubule-mediated delivery of the capsids to the nucleus [28].

\section{Viral replication and capsid assembly in the nucleus}

There is an emerging role for nuclear actin in transcription, chromatin remodeling, mRNA export and nuclear structure and integrity (reviewed in [29-31]). Likewise, nuclear actin might have a role in viral infection. Herpesvirus replication, gene expression and capsid formation take place within the host nucleus. Live imaging of movements of fluorescently labeled HSV-1 capsids in epithelial cell nuclei demonstrated that capsids undergo directed movements in the nucleus [32]. Capsid movement is both temperature and energy dependent. Using the actin inhibitor latrunculin A, Forest and colleagues concluded that these movements were dependent on actin [32]. In addition, the authors used the putative myosin inhibitor 


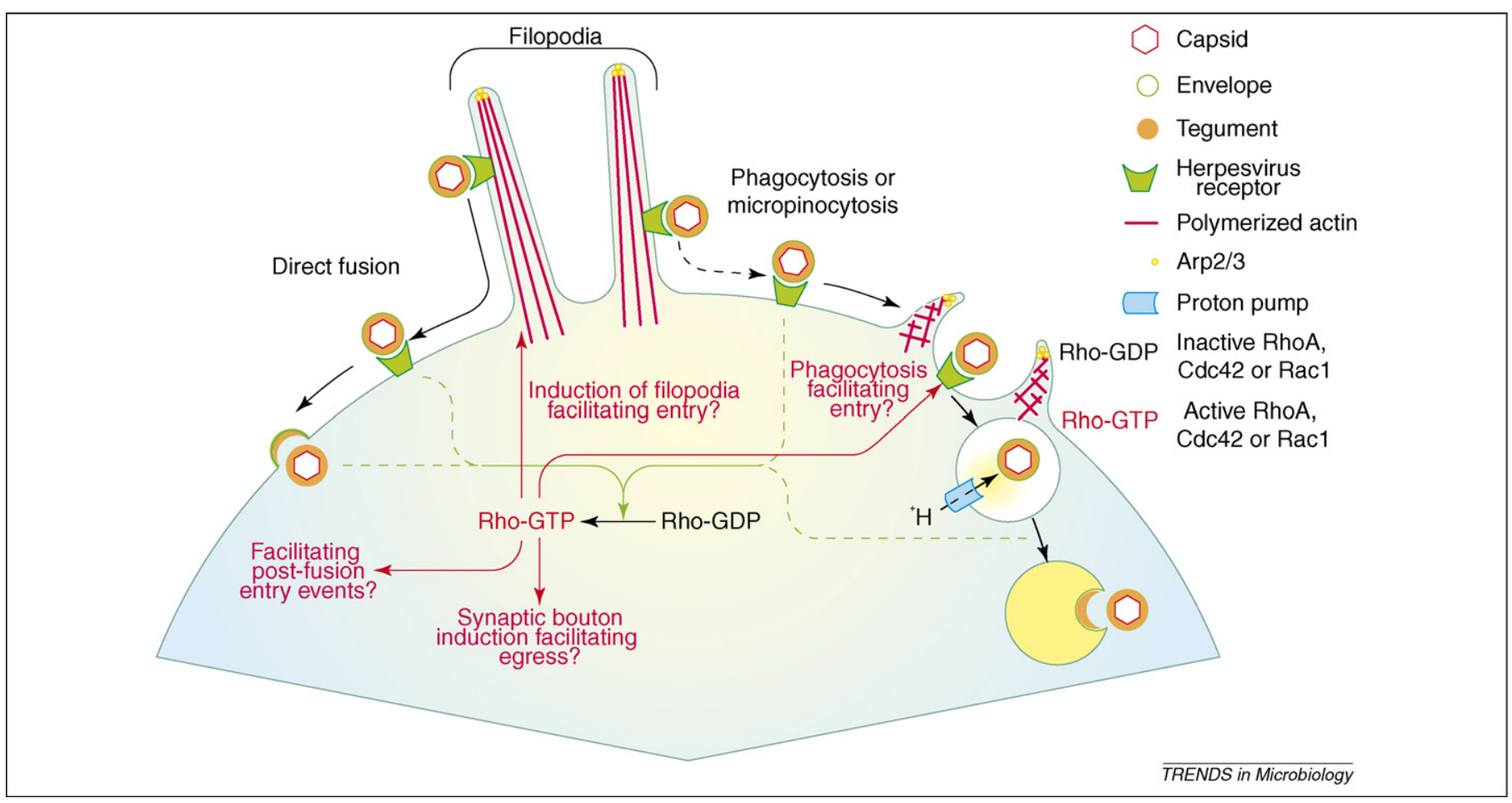

Figure 1. Involvement of actin and Rho GTPase signaling during herpesvirus entry. Herpesvirus particles associate with receptors on the cellular surface. This association is

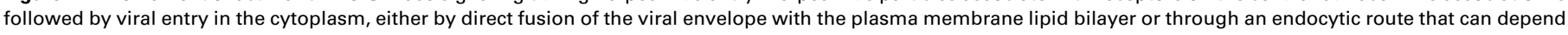

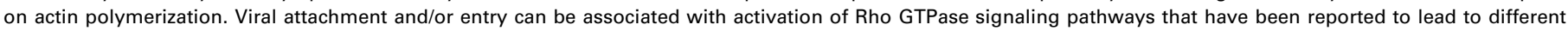
downstream effects, including formation of filopodia and formation of synaptic boutons. These events are cell-type specific.

2,3-butanedione monoxime (BDM) to show that the movements were myosin dependent. The authors speculated that capsids could be using actin for movement, either through actin-based polymerization or using the actin filaments as tracks.

\section{Herpesviruses induce the formation of actin filaments in the nucleus}

Using serial-section block-face scanning electron microscopy (SBFSEM), which enables the acquisition of volume electron-microscopic images, Feierbach et al. found that PRV capsids are associated with filamentous structures in the nuclei of infected neurons and epithelial cells [33]. Similar filaments associate with HSV-1 and HSV-2 nucleocapsids in infected central and peripheral neurons and glial cell nuclei [34]. Such filaments might be a conserved feature in alphaherpesvirus infections. Feierbach et al. found that actin filaments are formed in the nucleus of cells infected with PRV or HSV-1. Because these actin filaments associated with viral capsids during capsid assembly and were similar to the ones seen by SBFSEM [33], the authors concluded that intranuclear filaments are composed of actin. The function of nuclear actin during infection is currently unknown. The formation of higher order capsid assemblies is dependent on actin [32,33], but the addition of actin-depolymerizing drugs does not affect viral titers [35]. These seemingly conflicting results might be owing to differences in cell type or might simply reflect that higher order capsid structures are not essential for efficient viral assembly. In any case, further research will be necessary to elucidate the role (if any) of F-actin in capsid assembly.
Do nuclear actin filaments assist in intranuclear transport of herpes capsids (Figure 2)? Although a mechanism for capsid movement in the nucleus is still unknown, PRV capsids co-localize with the actin motor myosin $\mathrm{Va}$ in the nucleus [33]. Class V myosins are among the most thoroughly studied forms of unconventional myosins and considerable evidence supports their role in the transport of organelles and vesicles [36]. Myosin V is a two-headed myosin that shows progressive movement along actin filaments [36,37]. Myosin V is one of the fastest myosins, moving along actin filaments at a rate of $300-400 \mathrm{~nm} \mathrm{~s}^{-1}$ [38], which is comparable with the rate reported for directed movements of HSV-1 capsids [32]. Distinct from how Listeria and vaccinia virus use the actin cytoskeleton, herpesvirus capsids can move by myosin motors on nuclear actin filament tracks rather than by using actin polymerization-based movement (Figure 2).

\section{What triggers the formation of nuclear actin filaments during infection?}

Recent data from baculovirus suggest that the trigger might be a viral protein. Baculovirus-infected cells also form nuclear actin filaments at the time of virus assembly; these filaments co-localize with nucleocapsids and the baculovirus major capsid protein binds to F-actin [39]. The baculovirus protein p78/83 shares homology with WASP, a nucleator of actin, and promotes actin filament polymerization directly [40]. Herpesviruses do not seem to encode a homologous protein. Whether a herpesvirus protein(s) nucleates actin filaments directly or actin filament formation is an indirect response to infection remains to be seen. 


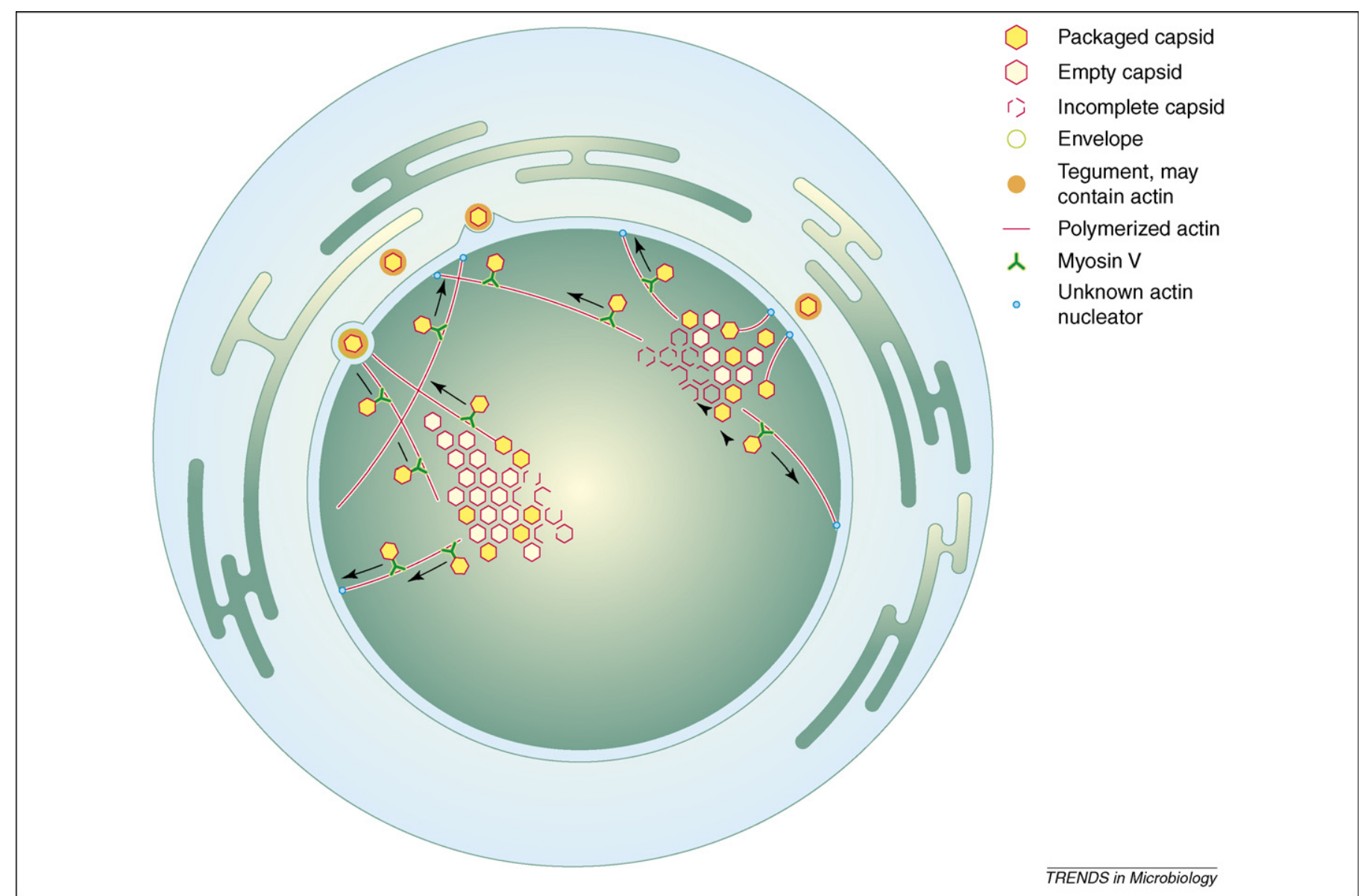

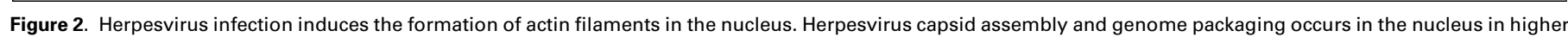

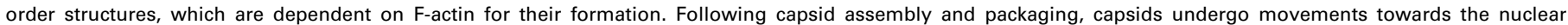
envelope, possibly along actin filaments through the motor myosin V. On exit from the nucleus, the virus might acquire actin as an integral part of its tegument.

\section{Actin is a component of the herpesvirus virion}

Recently, Baines and colleagues have conducted an elegant electron tomography study of HSV-1 undergoing envelopment at the inner nuclear membrane (INM) [41]. These images showed HSV-1 capsids connected to the INM by fibers $8-19 \mathrm{~nm}$ in length and $\leq 5 \mathrm{~nm}$ in width [41]. Fibers of similar dimensions bridged the virion envelope and the surface of the nucleocapsid in perinuclear virions. These authors speculated that the fibers are composed of actin owing to structural similarities between the fibers and F-actin [41]. Recent work has shown that host-derived actin is incorporated into the PRV virion and becomes an integral part of the outer tegument layer [42,43]. Virion-associated actin has been reported in other herpesviruses [44-49] and other enveloped viruses, including paramyxovirus, retrovirus and rhabdovirus [50-54]. But why do herpesviruses contain $\mathrm{F}$-actin in the virion? $\mathrm{F}$-actin could have a structural role or it might be a residual product of an earlier role, such as facilitating the association of the capsid with the INM (Figure 2). In any case, it is unclear where within the host cell virions acquire actin and if actin has any role in the virion.

\section{Herpesvirus egress from the nucleus}

Nuclear egress of alphaherpesvirus capsids involves engagement with the INM and subsequent budding into the perinuclear space through a tight meshwork of lamins and associated proteins [55,56]. Infection alters the nuclear structure through the reorganization of the lamin proteins on the INM [35,57-59]. Simpson-Holley and colleagues have demonstrated that actin is required for the characteristic nuclear expansion seen during HSV-1 infection of epithelial cells [35]. Emerin, a member of the LAP-2, emerin, MAN-1 (LEM) domain class of INM proteins, binds several nuclear components, including lamins, F-actin and nuclear myosin I. Emerin also enhances actin polymerization and might be involved in stabilizing actin at the nuclear membrane, thus contributing to nuclear integrity (reviewed in [60]). Recently, Morris and colleagues found that HSV-1 infection induces phosphorylation and delocalization of emerin, possibly resulting in changes in nuclear integrity [61]. Thus, herpesvirus infection might disrupt nuclear integrity during egress by the reorganization of INM proteins, possibly including nuclear actin.

\section{Virus envelopment and egress}

Role of actin in late stages of alphaherpesvirus infection As mentioned earlier, the physical connection of the herpesvirus entry receptor nectin with the actin cytoskeleton seems redundant for herpesvirus entry [18]. However, Sakisaka $e t a l$. found that it can facilitate viral spread from one cell to another [18]. Because alphaherpesvirus spread in polarized cells occurs through adherens junctions 


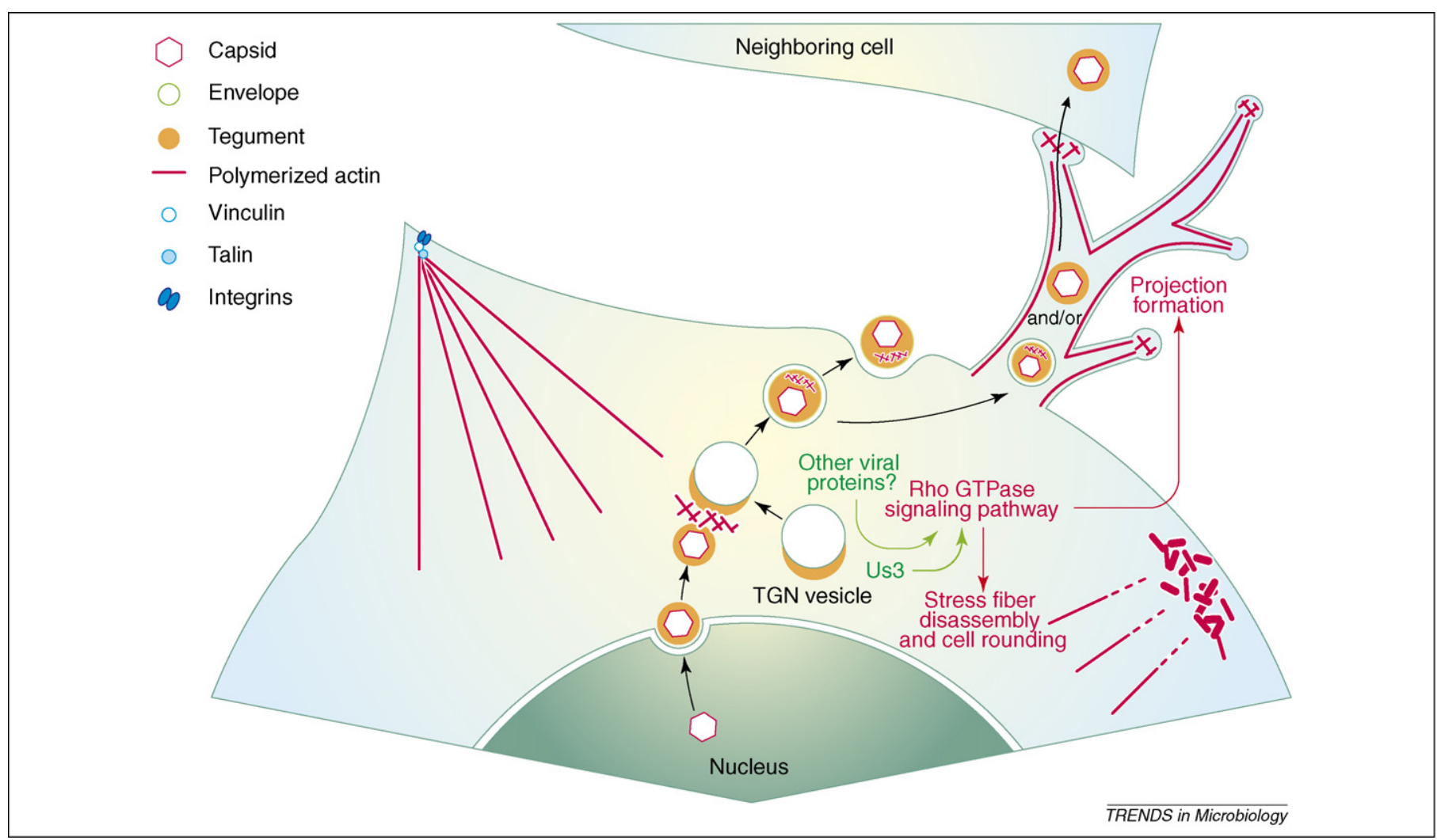

Figure 3. Involvement of actin and Rho GTPase signaling during herpesvirus egress. Actin incorporation in virus particles might occur at late stages of infection, during

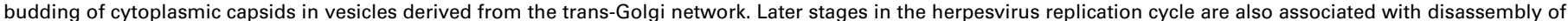

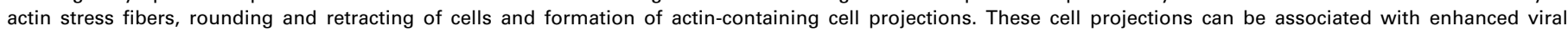
intercellular spread. Some of these events can be cell-type specific.

[62] and because afadin binding is required for nectin-1 to localize at cadherin-based adherens junctions [63], afadin might have a role in viral cell-to-cell spread by directing the gD receptor to the required cellular site in specific cell types. However, a role for the actin-binding properties of nectin during alphaherpesvirus spread seems to be cell-type dependent [64].

For PRV and MDV, for several cell types during infection, expression of the viral US3 serine/threonine kinase results in disassembly of actin stress fibers in different cell types [65,66] (Figure 3 ). The US3 protein is conserved among alphaherpesviruses and, although not essential for alphaherpesvirus replication, is implicated in several aspects of alphaherpesvirus infection, such as egress of progeny virus from the nucleus and protection of cells from apoptotic cell death [66-70]. PRV and MDV US3 are necessary and sufficient to induce stress fiber disassembly [65,66]. Transfection of HSV-2 US3 in HEp-2 cells also results in a loss of actin stress fibers, with a concomitant rounding of the cells [71]. PRV US3-induced disassembly of stress fibers was also associated with cell rounding and, in addition, with the induction of long, often branched actin-containing cell projections in sparsely seeded ST, RK13 and PK15 cells [72,73] (Figure 3). These cell projections established intimate contacts with adjacent cells and virus particles migrated in the projections towards the tip. Inhibition of US3-induced actin rearrangements using the actin-stabilizing drug jasplakinolide reduced antibody-resistant intercellular spread of PRV significantly, suggesting a role for this process in enhancing intercellular virus spread [73]. Although further experiments are needed to evaluate whether this mode of virus spread might occur under natural circumstances of infection, it is interesting that similar virusinduced cell projections have also been reported for HSV-1 in Vero and BHK cells and for VZV in HFF cells. It remains to be determined whether the US3 orthologs of these viruses are involved [74,75]. In addition, it is of interest that, similar to US3 of other alphaherpesviruses, the betaherpesvirus HCMV has also been shown to induce actin stress fiber disassembly, through the UL37 exon 1 protein, a process that has been reported to occur by $\mathrm{Ca}^{2+}$ release from the endoplasmic reticulum and/or alterations in mitochondrial bioenergetics [76-78].

\section{Interaction of alphaherpesviruses with Rho GTPase signaling during late stages of infection}

The exact mechanism of actin rearrangements by US3 and possibly other viral proteins remains largely unstudied, although it is likely that Rho GTPase signaling is involved. In support of this, co-expression of US3 with dominant active forms of $\mathrm{Cdc} 42$ and Rac1 inhibited HSV-2 US3-induced cell rounding and inhibition of RhoA signaling mimics the effects of US3 in cells infected with US3null PRV [71,73]. Our own data suggest that the kinase activity of PRV US3 is required for its ability to alter the actin cytoskeleton. It might therefore be that US3 phosphorylates and thereby (in)activates one or several components in the Rho GTPase signaling pathways. 
Of potential interest is that the poxvirus vaccinia virus interferes with Rho GTPase signaling to induce a very similar phenotype during infection: stress fiber disassembly, cell retraction and the formation of cell projections [79]. The F11L protein of vaccinia, which does not display kinase activity, is necessary and sufficient to induce these effects by directly binding and, thereby, inactivating RhoA. This finding indicates that two large DNA viruses have developed independent mechanisms to induce a similar phenotype, underscoring the importance of further investigating the significance of these actin rearrangements in the virus life cycle. Interestingly, the F11L-induced alterations in the actin cytoskeleton were associated with enhanced cell migration, possibly providing an additional means of efficient virus spread [79].

\section{Concluding remarks}

In summary, there is accumulating evidence that herpesviruses can usurp the actin cytoskeleton and actin-regulating Rho GTPase signaling pathways during the three major phases of herpesvirus infectious cycle: entry, replication and egress. However, many of the whys, wheres and hows still await further research: the biological significance of these events is, in several cases, not well understood, different herpesvirus interactions with actin and Rho GTPases seem to be cell-type dependent and, although several clues have been discovered about the underlying mechanisms, the exact network of viral and cellular proteins involved will undoubtedly turn out to be complex (Box 1). Unraveling the mechanistic details and biological significance of these interactions will tell us more about the cell biology of actin as well as provide new insights for herpesvirus biology.

Activation of Rho GTPases seems to be common during herpesvirus entry in a host cell but several of the mechanistic details await discovery $[12,19,22]$. For example, what are the particular events during herpesvirus entry responsible for Rho GTPase signaling? There is some evidence that the interaction between the $\mathrm{gD}$ envelope protein and nectins might lead to Cdc42- and perhaps Rac- and RhoAdriven processes [12,19]. However, interactions between herpesviruses and host cells during viral entry are complex and involve different viral ligands and different cellular receptors [15]. Do other gD receptors and/or other viral ligand-receptor interactions lead to the activation of Rho GTPases? Nectin-mediated Rho GTPase activation during alphaherpesvirus entry results in synaptic bouton formation in neurons and filopodia induction in $\mathrm{CHO}$ cells,

\section{Box 1. Issues for the future}

- Role of gD-nectin and possibly other viral ligand-receptor interactions in Rho GTPase signaling and actin dynamics during entry and their biological significance

- Biology underlying the cell-type-dependent role for actin in entry

- Role of actin and myosin $\mathrm{V}$ in herpesvirus assembly in the nucleus and egress from the nucleus

- Viral and cellular factors involved in actin filament induction in the nucleus

- Origin and function of F-actin in the herpesvirus virion tegument

- Role of US3 and other viral and cellular proteins in actin rearrangements late in infection and their biological significance suggesting roles in enhancing egress of progeny virus and viral uptake, respectively $[12,19]$. Although providing a first clue, there is a need for further elaboration of the biological significance of Rho GTPase activation and actin reorganization during virus entry.

The role of actin during entry relies heavily on the particular cell type that becomes infected. We suggest that the mode of entry might be one of the factors involved in this cell-type dependence. Entry by direct fusion at the plasma membrane seems to be independent of actin [9], whereas entry by one or more of the recently described endocytic routes of entry might rely more heavily on actin and Rho GTPase signaling pathways [12].

The actin cytoskeleton and associated proteins are also implicated in herpesvirus capsid assembly and nuclear egress [32,33,41]. Capsid assembly and genome packaging occurs in the nucleus in higher order structures that might depend on F-actin for their formation [33]. Many questions remain unanswered. For example, we do not understand how actin filament formation occurs after infection. Viralencoded proteins might nucleate the filaments or perhaps filament formation is a host response to infection. Similarly, we are in the early stages of understanding how capsids are directed from their site of formation to sites of egress at the nuclear membrane. It is probable that they move on actin filaments using the motor protein myosin $\mathrm{V}$ but little is known about the process [32,33]. In addition, herpesviruses might need to reorganize the actin meshwork at the INM for nuclear egress. Herpesvirus interaction with emerin might facilitate this process [61].

Later stages of the herpesvirus replication cycle have been associated with disassembly of actin stress fibers, retraction and rounding of the cells and the formation of actin-containing cell projections. The US3 protein, a nonessential viral kinase conserved among alphaherpesviruses, has a key role in these processes [65,66,71-73]. One idea is that US3 interferes with Rho GTPase signaling, although this needs further analysis [71,73]. The US3-induced actin rearrangements have been associated with enhanced intercellular virus spread in sparsely seeded cells [73]. It will be interesting to further elucidate the biological significance and underlying mechanism of US3-induced actin rearrangements. This research is complicated by the fact that US3, although not essential for viral replication, is implicated in several other important aspects of the viral life cycle, including nuclear egress of progeny virus and protection of cells from apoptotic cell death [66-70].

\section{Acknowledgements}

We thank Silvia Piccinotti for the drawings used in this review. L.E. and B.F. are supported by the National Institute of Neurological Disorders and Stroke (NIH-NINDS; grant R01 33506) and the Dana Research Foundation. H.F. is supported by research grants from the Research Foundation - Flanders (F.W.O. Vlaanderen, grant nr. G.0196.06) and a Concerted Research Action fund of Ghent University. We thank all the present and past members of the Favoreel research group and the members of the Enquist laboratory. In particular, we would like to mention all the hard work of Geert Van Minnebruggen, Nick De Regge, Céline Van den Broeke, Matthias Deruelle, Sarah Glorieux and Silvia Piccinotti related to this topic. We also thank Hans Nauwynck for many fruitful discussions. We apologize to any colleagues whose work we were unable to mention because of space constraints. 


\section{References}

1 Etienne-Manneville, S. and Hall, A. (2002) Rho GTPases in cell biology. Nature 420, 629-635

2 Vignal, E. et al., eds (2003) Signaling Networks of Rho GTPases, R.G. Landes Co.

3 Schwartz, M. (2004) Rho signalling at a glance. J. Cell Sci. 117, 54575458

4 Stevens, J.M. et al. (2006) Actin-dependent movement of bacterial pathogens. Nat. Rev. Microbiol. 4, 91-101

5 Gouin, E. et al. (2005) Actin-based motility of intracellular pathogens. Curr. Opin. Microbiol. 8, 35-45

6 Smith, G.A. and Enquist, L.W. (2002) Break ins and break outs: viral interactions with the cytoskeleton of mammalian cells. Annu. Rev. Cell Dev. Biol. 18, 135-161

7 Dohner, K. et al. (2005) Viral stop-and-go along microtubules: taking a ride with dynein and kinesins. Trends Microbiol. 13, 320-327

8 Koyama, A.H. and Uchida, T. (1987) The mode of entry of herpes simplex virus type 1 into Vero cells. Microbiol. Immunol. 31, 123-130

9 Sodeik, B. et al. (1997) Microtubule-mediated transport of incoming herpes simplex virus 1 capsids to the nucleus. J. Cell Biol. 136, 1007-1021

10 Rue, C.A. and Ryan, P. (2003) A role for glycoprotein C in pseudorabies virus entry that is independent of virus attachment to heparan sulfate and which involves the actin cytoskeleton. Virology 307, 12-21

11 Finnen, R.L. et al. (2006) Postentry events are responsible for restriction of productive varicella-zoster virus infection in Chinese hamster ovary cells. J. Virol. 80, 10325-10334

12 Clement, C. et al. (2006) A novel role for phagocytosis-like uptake in herpes simplex virus entry. J. Cell Biol. 174, 1009-1021

13 Milne, R.S. et al. (2005) Glycoprotein D receptor-dependent, low-pHindependent endocytic entry of herpes simplex virus type 1. J. Virol. 79, $6655-6663$

14 Nicola, A.V. et al. (2003) Roles for endocytosis and low $\mathrm{pH}$ in herpes simplex virus entry into $\mathrm{HeLa}$ and Chinese hamster ovary cells. J. Virol. 77, 5324-5332

15 Spear, P.G. (2004) Herpes simplex virus: receptors and ligands for cell entry. Cell. Microbiol. 6, 401-410

16 Kawakatsu, T. et al. (2002) Trans-interactions of nectins induce formation of filopodia and lamellipodia through the respective activation of Cdc42 and Rac small G proteins. J. Biol. Chem. 277, 50749-50755

17 Mizoguchi, A. et al. (2002) Nectin: an adhesion molecule involved in formation of synapses. J. Cell Biol. 156, 555-565

18 Sakisaka, T. et al. (2001) Requirement of interaction of nectin- $1 \alpha / \mathrm{HveC}$ with afadin for efficient cell-cell spread of herpes simplex virus type 1 . J. Virol. 75, 4734-4743

19 De Regge, N. et al. (2006) Alpha-herpesvirus glycoprotein D interaction with sensory neurons triggers formation of varicosities that serve as virus exit sites. J. Cell Biol. 174, 267-275

20 Ch'ng, T.H. and Enquist, L.W. (2005) Neuron-to-cell spread of pseudorabies virus in a compartmented neuronal culture system. J. Virol. 79, 10875-10889

21 Saksena, M.M. et al. (2006) Herpes simplex virus type 1 accumulation, envelopment, and exit in growth cones and varicosities in mid-distal regions of axons. J. Virol. 80, 3592-3606

22 Hoppe, S. et al. (2006) Early herpes simplex virus type 1 infection is dependent on regulated Rac1/Cdc42 signalling in epithelial MDCKII cells. J. Gen. Virol. 87, 3483-3494

23 Lehmann, M.J. et al. (2005) Actin- and myosin-driven movement of viruses along filopodia precedes their entry into cells. J. Cell Biol. 170, $317-325$

24 Nicola, A.V. et al. (2005) Herpes simplex virus type 1 enters human epidermal keratinocytes, but not neurons, via a $\mathrm{pH}$-dependent endocytic pathway. J. Virol. 79, 7609-7616

25 Welch, M.D. and Mullins, R.D. (2002) Cellular control of actin nucleation. Annu. Rev. Cell Dev. Biol. 18, 247-288

26 Naranatt, P.P. et al. (2003) Kaposi's sarcoma-associated herpesvirus induces the phosphatidylinositol 3-kinase-PKC- $\zeta-M E K-E R K$ signaling pathway in target cells early during infection: implications for infectivity. J. Virol. 77, 1524-1539

27 Sharma-Walia, N.et al. (2004) Kaposi's sarcoma-associated herpesvirus/ human herpesvirus 8 envelope glycoprotein $\mathrm{gB}$ induces the integrin-dependent focal adhesion kinase-Src-phosphatidylinositol 3-kinase-rho GTPase signal pathways and cytoskeletal rearrangements. J. Virol. 78, 4207-4223

28 Naranatt, P.P. et al. (2005) Kaposi's sarcoma-associated herpesvirus modulates microtubule dynamics via RhoA-GTP-diaphanous 2 signaling and utilizes the dynein motors to deliver its DNA to the nucleus. J. Virol. 79, 1191-1206

29 de Lanerolle, P. et al. (2005) Actin and myosin I in the nucleus: what next? Nat. Struct. Mol. Biol. 12, 742-746

30 Pederson, T. and Aebi, U. (2005) Nuclear actin extends, with no contraction in sight. Mol. Biol. Cell 16, 5055-5060

31 Bettinger, B.T. et al. (2004) Actin up in the nucleus. Nat. Rev. Mol. Cell Biol. 5, 410-415

32 Forest, T. et al. (2005) Active intranuclear movement of herpesvirus capsids. Nat. Cell Biol. 7, 429-431

33 Feierbach, B. et al. (2006) Alpha-herpesvirus infection induces the formation of nuclear actin filaments. PLoS Pathog. 2, e85

34 Ecob-Johnston, M.S. and Whetsell, W.O., Jr (1979) Host-cell response to herpes virus infection in central and peripheral nervous tissue in vitro. J. Gen. Virol. 44, 747-757

35 Simpson-Holley, M. et al. (2005) Identification and functional evaluation of cellular and viral factors involved in the alteration of nuclear architecture during herpes simplex virus 1 infection. J. Virol. $79,12840-12851$

36 Bridgman, P.C. (2004) Myosin-dependent transport in neurons. J. Neurobiol. 58, 164-174

37 Mehta, A.D. et al. (1999) Myosin-V is a processive actin-based motor. Nature 400, 590-593

38 Cheney, R.E. et al. (1993) Brain myosin-V is a two-headed unconventional myosin with motor activity. Cell 75, 13-23

39 Charlton, C.A. and Volkman, L.E. (1991) Sequential rearrangement and nuclear polymerization of actin in baculovirus-infected Spodoptera frugiperda cells. J. Virol. 65, 1219-1227

40 Goley, E.D. et al. (2006) Dynamic nuclear actin assembly by Arp2/3 complex and a baculovirus WASP-like protein. Science 314, 464-467

41 Baines, J.D. et al. (2007) Electron tomography of nascent herpes simplex virus virions. J. Virol. 81, 2726-2735

42 del Rio, T. et al. (2005) Actin is a component of the compensation mechanism in pseudorabies virus virions lacking the major tegument protein VP22. J. Virol. 79, 8614-8619

43 Michael, K. et al. (2006) Composition of pseudorabies virus particles lacking tegument protein US3, UL47, or UL49 or envelope glycoprotein E. J. Virol. 80, 1332-1339

44 Baldick, C.J., Jr and Shenk, T. (1996) Proteins associated with purified human cytomegalovirus particles. J. Virol. 70, 6097-6105

45 Grunewald, K. et al. (2003) Three-dimensional structure of herpes simplex virus from cryo-electron tomography. Science 302, 1396-1398

46 Kattenhorn, L.M. et al. (2004) Identification of proteins associated with murine cytomegalovirus virions. J. Virol. 78, 11187-11197

47 Wong, M.L. and Chen, C.H. (1998) Evidence for the internal location of actin in the pseudorabies virion. Virus Res. 56, 191-197

48 Varnum, S.M. et al. (2004) Identification of proteins in human cytomegalovirus (HCMV) particles: the HCMV proteome. J. Virol. 78, 10960-10966

49 Zhu, F.X. et al. (2005) Virion proteins of Kaposi's sarcoma-associated herpesvirus. J. Virol. 79, 800-811

50 Bohn, W. et al. (1987) Replica-immunogold technique applied to studies on measles virus morphogenesis. Scanning Microsc. 1, 319-330

51 Naito, S. and Matsumoto, S. (1978) Identification of cellular actin within the rabies virus. Virology 91, 151-163

52 Ott, D.E. et al. (1996) Cytoskeletal proteins inside human immunodeficiency virus type 1 virions. J. Virol. 70, 7734-7743

53 Sagara, J. et al. (1995) Cellular actin-binding ezrin-radixin-moesin (ERM) family proteins are incorporated into the rabies virion and closely associated with viral envelope proteins in the cell. Virology 206, 485-494

54 Vainiopaa, R. et al. (1978) Measles virus polypeptides in purified virions and in infected cells. Acta Pathol. Microbiol. Scand. [B] 86B, 379-385

55 Skepper, J.N. et al. (2001) Herpes simplex virus nucleocapsids mature to progeny virions by an envelopment $\rightarrow$ deenvelopment $\rightarrow$ reenvelopment pathway. J. Virol. 75, 5697-5702

56 Mettenleiter, T.C. (2002) Herpesvirus assembly and egress. J. Virol. $76,1537-1547$ 
57 Scott, E.S. and O'Hare, P. (2001) Fate of the inner nuclear membrane protein lamin B receptor and nuclear lamins in herpes simplex virus type 1 infection. J. Virol. 75, 8818-8830

58 Reynolds, A.E. et al. (2004) Conformational changes in the nuclear lamina induced by herpes simplex virus type 1 require genes $U(L) 31$ and $U(L) 34$. J. Virol. 78, 5564-5575

59 Muranyi, W. et al. (2002) Cytomegalovirus recruitment of cellular kinases to dissolve the nuclear lamina. Science 297, 854-857

60 Bengtsson, L. and Wilson, K.L. (2004) Multiple and surprising new functions for emerin, a nuclear membrane protein. Curr. Opin. Cell Biol. 16, 73-79

61 Morris, J. et al. (2007) HSV infection induces phosphorylation and delocalisation of Emerin, a key inner nuclear membrane protein. J. Virol. DOI: 10.1128/JVI.02354-06

62 Johnson, D.C. and Huber, M.T. (2002) Directed egress of animal viruses promotes cell-to-cell spread. J. Virol. 76, 1-8

63 Takahashi, K. et al. (1999) Nectin/PRR: an immunoglobulin-like cell adhesion molecule recruited to cadherin-based adherens junctions through interaction with Afadin, a PDZ domain-containing protein. J. Cell Biol. 145, 539-549

64 Krummenacher, C. et al. (2003) Cellular localization of nectin-1 and glycoprotein D during herpes simplex virus infection. J. Virol. 77, 8985-8999

65 Van Minnebruggen, G. et al. (2003) Pseudorabies virus US3 protein kinase mediates actin stress fiber breakdown. J. Virol. 77, 9074-9080

66 Schumacher, D. et al. (2005) The protein encoded by the US3 orthologue of Marek's disease virus is required for efficient deenvelopment of perinuclear virions and involved in actin stress fiber breakdown. J. Virol. 79, 3987-3997

67 Wagenaar, F. et al. (1995) The US3-encoded protein kinase from pseudorabies virus affects egress of virions from the nucleus. J. Gen. Virol. 76, 1851-1859

68 Leopardi, R. et al. (1997) The herpes simplex virus 1 protein kinase US3 is required for protection from apoptosis induced by the virus. Proc. Natl. Acad. Sci. U. S. A. 94, 7891-7896
69 Ogg, P.D. et al. (2004) The HSV-1 Us3 protein kinase is sufficient to block apoptosis induced by overexpression of a variety of Bcl-2 family members. Virology 319, 212-224

70 Geenen, K. et al. (2005) The pseudorabies virus US3 protein kinase possesses anti-apoptotic activity that protects cells from apoptosis during infection and after treatment with sorbitol or staurosporine. Virology 331, 144-150

71 Murata, T. et al. (2000) Expression of herpes simplex virus type 2 US3 affects the $\mathrm{Cdc} 42 / \mathrm{Rac}$ pathway and attenuates c-Jun N-terminal kinase activation. Genes Cells 5, 1017-1027

72 Calton, C.M. et al. (2004) The pseudorabies virus serine/threonine kinase Us3 contains mitochondrial, nuclear and membrane localization signals. Virus Genes 29, 131-145

73 Favoreel, H.W. et al. (2005) Cytoskeletal rearrangements and cell extensions induced by the US3 kinase of an alphaherpesvirus are associated with enhanced spread. Proc. Natl. Acad. Sci. U. S. A. 102, 8990-8995

74 van Leeuwen, H. et al. (2002) Evidence of a role for nonmuscle myosin II in herpes simplex virus type 1 egress. J. Virol. 76, 3471-3481

75 Zapata, H.J. et al. (2007) Varicella-zoster virus infection of human fibroblast cells activates the c-Jun $\mathrm{N}$-terminal kinase pathway. J. Virol. 81, 977-990

76 Poncet, D. et al. (2006) Cytopathic effects of the cytomegalovirusencoded apoptosis inhibitory protein vMIA. J. Cell Biol. 174, 985-996

77 Sharon-Friling, R. et al. (2006) Human cytomegalovirus pUL37x1 induces the release of endoplasmic reticulum calcium stores. Proc. Natl. Acad. Sci. U. S. A. 103, 19117-19122

78 Bentz, G.L. et al. (2006) Human cytomegalovirus (HCMV) infection of endothelial cells promotes naive monocyte extravasation and transfer of productive virus to enhance hematogenous dissemination of HCMV. J. Virol. 80, 11539-11555

79 Valderrama, F. et al. (2006) Vaccinia virus-induced cell motility requires F11L-mediated inhibition of RhoA signaling. Science 311, $377-381$

\section{Reproduction of material from Elsevier articles}

Interested in reproducing part or all of an article published by Elsevier, or one of our article figures? If so, please contact our Global Rights Department with details of how and where the requested material will be used. To submit a permission request online, please contact:

Elsevier

Global Rights Department

PO Box 800

Oxford OX5 1DX, UK

Phone: $+44(0) 1865843830$

Fax: +44 (0)1865 853333

permissions@elsevier.com

Alternatively, please visit:

\section{www.elsevier.com/locate/permissions}

